\title{
GRADE-III SLIPPED CAPITAL FEMORAL EPIPHYSIS WITH UNSTABILITY: A REPORT OF THREE CASES
}

\author{
Pavel Šponer, Karel Urban \\ Charles University in Prague, Faculty of Medicine and University Hospital in Hradec Králové, Czech Republic: \\ Department of Orthopaedic Surgery
}

Summary: We reviewed the cases of three patients with an unstable grade-III slipped capital femoral epiphysis treated between 2001 and 2003. Clinical records and imaging studies were reviewed for patient's history, anatomic features of the slip, definitive treatment and clinical outcome. The duration of the follow-up ranged from twenty-four to fourty-eight months.

Key words: Slipped capital femoral epiphysis; In situ stabilization; Femoral osteotomy

\section{Introduction}

The newer clinical classification of slipped capital femoral epiphysis reported by Loder depends on the ability of the child to walk (8). He classified a slip as unstable if the child had such severe pain that walking was not possible even with crutches, regardless of the duration of the symptoms. The slip was classified as stable if walking and weight-bearing were still possible with or without crutches. Unstable slipped capital femoral epiphyses have a much higher prevalence of avascular necrosis compared with stable slipped capital femoral epiphyses. The treatment of an unstable slipped capital femoral epiphysis is controversial, particullary the role of preoperative traction and timing of reduction $(2,8,9)$.

We are presenting the cases of three patients who had severe slipped capital femoral epiphysis with instability to raise attention to this rare but potentially serious disorder affecting adolescents. In addition, we evaluated the clinical effectiveness of our treatment.

\section{Case reports}

\section{CASE 1}

A fourteen-year-old boy had severe left hip pain following turning over in bed, without prior hip symptoms. There were six hours from the onset of the symptoms to the arrival to hospital. An external rotation deformity, shortening and marked limitation of motion secondary to pain were present on physical examination. The pain was severe enough to prevent weight-bearing. Radiographs demonstrated an inferior and posterior severe slip of the left proximal femoral epiphysis relative to the metaphysis, without signs of superior and anterior remodeling on the femoral metaphysis. The Southwick angle measured 75 degrees.

Immediately skin traction was used for acute severe unstable slipped capital femoral epiphysis. The calcium and phosphate levels in the serum were measured and being normal. Extreme hypercalciury fU-Ca 21,46 mmol/day (normal $<4,00 \mathrm{mmol} /$ day) and high phosphatury fU-P 59,6 $\mathrm{mmol} /$ day (normal $<45 \mathrm{mmol} /$ day) were found. Aminoacidury and T3,T4,TSH levels were tested and early operative management was contraindicated due to the metabolic disorder. With relief of pelvifemoral muscle spasm during four weeks of traction, no gradual reducing of epiphysis has been obtained. The patient was allowed to be ambulatory. Osteopenia of the relative vitamin D and calcium deficiency in the pubertal rapid growth spurt age was treated with administration of vitamin D daily in therapeutic doses $(2800$ I.U.). Avascular necrosis developed 6 months after initial trivial traumatic episode. Full correction of a metabolic disorder was obtained after 18 months. Intertrochanteric femoral osteotomy with flexion, abduction and internal rotation of the distal fragment was planned and performed.

At two years postoperatively, the left hip had excellent range of motion, an absence of pain. The patient had no limp in spite of mild limb-length discrepancy.

\section{CASE 2}

An eleven-year-old girl presented with an one-week history of pain in the right hip. The medical history, including that for endocrine disorders, was negative. Initially, the pain had been mild, but three days before the patient presented at our institution, the symptoms began to worsen and the girl could not walk. Physical examination revealed marked limitation of flexion, abduction and internal rotation, pain 


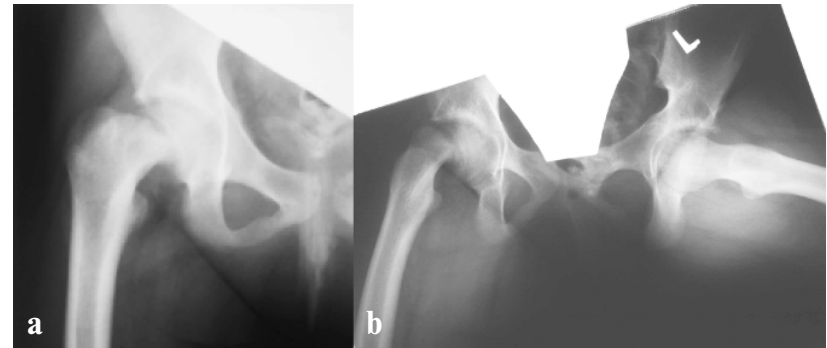

Fig. 1 a,b: Anteroposterior radiogram of the right hip (a), frog-leg radiogram of both hips (b).

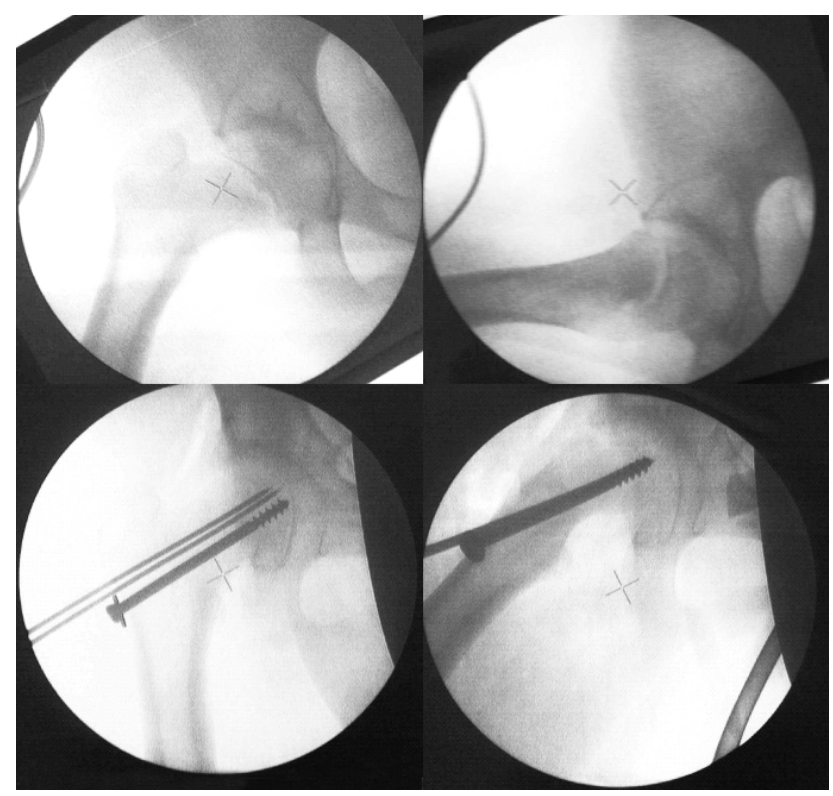

Fig. 2 a-d: Closed partial reduction $(a, b)$ and internal fixation $(c, d)$ of the right capital femoral epiphysis.

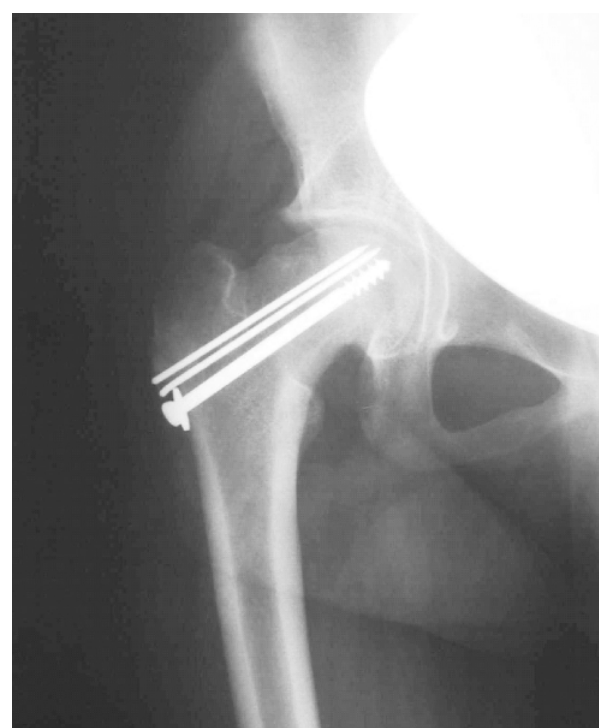

Fig. 3: The capital femoral physis is closed eight months after surgery. with motion. Radiographs of both hips demonstrated a severe slip of the right hip according to Boyer in which the Southwick angle measured 61 degrees (Fig. 1).

The patient was diagnosed with an acute severe unstable slipped capital femoral epiphysis. A closed partial (nonanatomic) reduction was performed less than twenty-four hours after presentation and internal fixation with use of one screw and 2 Kirschner wires was applied (Fig. 2). The positions of the screw and Kirschner wires were measured on the postoperative radiographs by method of Mulholland and Gunn in which the screw-position score was 1.7. The distance from the tip of the screw or Kirschner wire to the subchondral bone measured $4 \mathrm{~mm}$ in the first Kirschner wire and more than $5 \mathrm{~mm}$ in the second Kirschner wire and screw (Fig. 3).

At thirty-six months of follow-up, there was no pain, the patient had only limitation of internal rotation of the involved hip and a mild limp without presence of osteonecrosis (Fig. 4).

\section{CASE 3}

A twelve-year-old girl presented to us with the one-week history of pain in the left hip. Two years ago the patient underwent in situ stabilization of the right slipped capital femoral epiphysis using two screws. The medical history, including that for endocrine diseases, was negative. Radiographs demonstrated a mild slip of the left capital femoral epiphysis. Immediately preoperative traction was used to release pelvifemoral muscle spasm and in situ stabilization was planned. At sixth day of traction the girl suffered severe left hip pain following turning over in bed. Additional displacement occurred after the initial diagnosis, the capital femoral epiphysis became severely slipped and thus the Southwick angle reached 55 degrees.

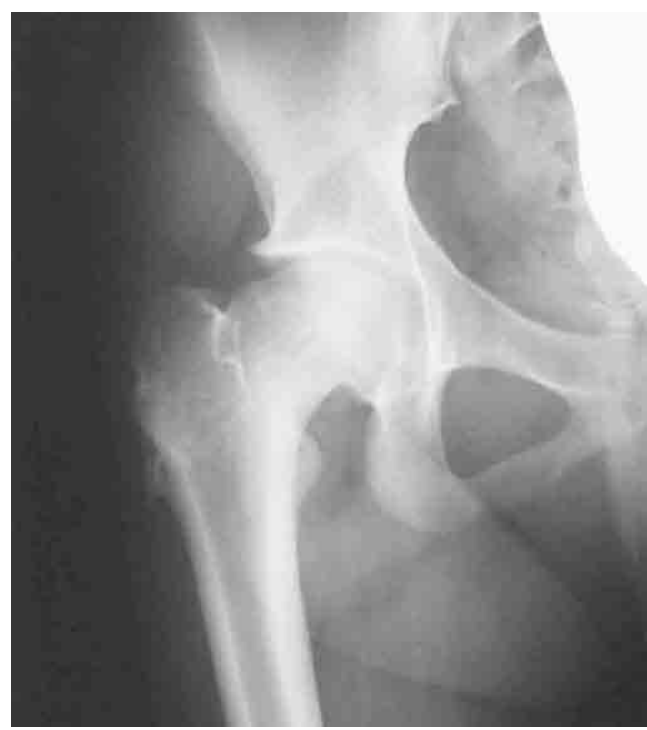

Fig. 4: Radiogram after removal of the pins. 
A closed partial (nonanatomic) reduction was performed less than twenty-four hours after additional displacement and internal fixation using two screws was done. The screw-position score was 1 . The distance from the tip of the screw to the subchondral bone measured $2 \mathrm{~mm}$ in the first screw and $1 \mathrm{~mm}$ in the second one.

At nine months follow-up, there was left groin pain with a loss of motion of the hip, particularly internal rotation. Radiographs revealed changes significant for avascular necrosis and closed capital femoral physis without protrusion of internal fixation device. The screws were removed and avascular necrosis was treated conservatively. Nonweight-bearing walking with crutches, range-of-motion exercises and anti-inflammatory medication helped to minimize symptoms. At two years postoperatively, the patient had limitation of internal rotation in the involved hip and a mild limp with groin pain. Intertrochanteric femoral osteotomy with flexion, abduction and internal rotation of the distal fragment was planned and performed to diminish subjective complaints.

\section{Discussion}

Unstable slip should be managed as an emergency and the patient ought to be admitted to a hospital immediately. Bilateral split Russell traction with medial rotation straps on the affected thigh and leg or simple skin traction for few days were recommended to decrease the risk of avascular necrosis by gradually absorbing the inflammatory effusion associated with an acute slip and gradually reducing the epiphysis (3). Preliminary longitudinal - medial rotational traction was ineffective in reduction of unstable slipped capital femoral epiphysis in our case 1 . If reduction was not achieved by skin traction, closed manipulative partial reduction under general anesthesia was reported $(1,4)$. Nowadays the reduction of the unstable slip occured with the induction of anesthesia and the position of the patient on the operating table is preferred (8). Controversy over the immediate and late closed reduction continues $(2,8,9)$. After reduction (no attempt of closed reduction if more than three weeks have elapsed since the acute episode), the capital femoral epiphysis with accepted residual deformity is temporarily fixed with two guide wires prior to drilling and tapping to prevent any risk of rotation or distraction of the epiphysis. The second screw should be inserted to enhance stability after insertion the first cannulated screw if deemed necessary $(5,6,7)$.

Osteonecrosis is a serious complication of the treatment of slipped capital femoral epiphysis and all cases are radio- graphically apparent within one year after slipped capital femoral epiphysis $(8,10)$. Primary injury to the epiphyseal vasculature having occured at the time of the acute slipped capital femoral epiphysis is well documented in case 1 . Non-weight-bearing walking with crutches, range-of-motion exercises and anti-inflammatory medication can minimize symptoms and epiphyseal collapse. If there is pin protrusion and the physis is closed, removing the pin helps to reduce additional damage to the hip joint. Hip arthrodesis or arthroplasty may be necessary in severe cases. Unstable slips characterized by the patient's inability to bear weight, intraarticular effusion and epiphyseal instability on the femoral neck are at much higher risk for development of avascular necrosis. Families must be informed of this increased risk.

\section{References}

1. Casey BH, Hamilton HW, Bobechko WP. Reduction of acutely slipped upper femoral epiphysis. J Bone Joint Surg 1972: 54-B: 607-14.

2. De Sanctis A, Di Gennaro G, Pempinello C, Della Corte S, Carannante G. Is gentle manipulative reduction and percutaneous fixation with a single screw the best management of acute and acute-on-chronic slipped capital femoral epiphysis? A report of 70 patients. J Pediat Orthop Part B 1996: 5: 90-5.

3. Dietz FR. Traction reduction of acute and acute-on-chronic slipped capital femoral epiphysis. Clin Orthop 1994: 302: 101-10.

4. Fahey JJ, O’Brien ET. Acute slipped capital femoral epiphysis: review of the literature and report of ten cases. J Bone Joint Surg 1965: 47-A: 1105-27.

5. Herman MJ, Dormans JP, Davidson RS, Drummond DS, Gregg JR. Screw fixation of grade III slipped capital femoral epiphysis. Clin Orthop 1996: 322: 77-85.

6. Karol LA, Doane RM, Cornicelli SF, Zak PA, Haut RC, Manoli A. Single versus double screw fixation for treatment of slipped capital femoral epiphysis: a biomechanical analysis. J Pediatr Orthop 1992: 12: 741-45.

7. Kibiloski LJ, Doane RM, Karol LA, Haut RC, Loder RT. Biomechanical analysis of single- versus double-screw fixation in slipped capital femoral epiphysis at physiological load levels. J Pediatr Orthop 1994: 14: 627-30.

8. Loder RT, Richards BS, Shapiro PS, Reznick LR, Aronson DD. Acute slipped capital femoral epiphysis: the importance of physeal stability. J Bone Joint Surg 1993: 75-A: 1134-40.

9. Peterson MD, Weiner DS, Green NE, Terry CL. Acute slipped capital femora epiphysis: the value and safety of urgent manipulative reduction. J Pediat Orthop 1997: 17: 648-54.

10. Tokmakova KP, Stanton RP, Mason DE. Factors influencing the development of osteonecrosis in patients treated for slipped capital femoral epiphysis. J Bone Joint Surg 2003: 85-A: 798-801.

Submitted September 2005.

Accepted March 2006.

MUDr. Pavel Šponer,

University Hospital in Hradec Králové,

Department of Orthopaedic Surgery,

Sokolská 581,

50005 Hradec Králové,

Czech Republic.

e-mail: sponer.p@seznam.cz 